\title{
Practical calculation method to estimate the absolute boron concentration in tissues using ${ }^{18}$ F-FBPA PET
}

\author{
Tadashi Watabe $^{1,2} \cdot$ Kohei Hanaoka ${ }^{1}$ Sadahiro Naka ${ }^{4}$ Yasukazu Kanai ${ }^{2,3}$. \\ Hayato Ikeda $^{1} \cdot$ Masanao Aoki $^{1} \cdot$ Eku Shimosegawa ${ }^{1,2,3} \cdot$ Mitsunori Kirihata $^{5}$. \\ Jun Hatazawa ${ }^{1,2,6}$
}

Received: 21 March 2017/ Accepted: 16 April 2017/Published online: 24 April 2017

(c) The Author(s) 2017. This article is an open access publication

\begin{abstract}
Purpose The purpose of this study was to establish a practical method to estimate the absolute boron concentrations in the tissues based on the standardized uptake values (SUVs) after administration of 4-borono-phenylalanine (BPA) using 4-borono-2- ${ }^{18}$ F-fluoro-phenylalanine $\left({ }^{18} \mathrm{~F}\right.$-FBPA) PET.

Methods Rat xenograft models of C6 glioma ( $n=7$, body weight $241 \pm 28.0 \mathrm{~g}$ ) were used for the study. PET was performed $60 \mathrm{~min}$ after intravenous injection of ${ }^{18} \mathrm{~F}-\mathrm{FBPA}$ $(30.5 \pm 0.7 \mathrm{MBq})$. After the PET scanning, BPA-fructose $(167.3 \pm 18.65 \mathrm{mg} / \mathrm{kg})$ was administered by slow intravenous injection to the same subjects. The rats were killed 60 min after the BPA injection and tissue samples were collected from the major organs and tumors. The absolute boron concentrations (unit: $\mathrm{ppm}$ ) in the samples were measured by inductively coupled plasma optical emission spectrometry (ICP-OES). The boron concentrations in the
\end{abstract}

Electronic supplementary material The online version of this article (doi:10.1007/s12149-017-1172-5) contains supplementary material, which is available to authorized users.

Tadashi Watabe

watabe@tracer.med.osaka-u.ac.jp

1 Department of Nuclear Medicine and Tracer Kinetics, Osaka University Graduate School of Medicine, 2-2 Yamadaoka, Suita, Osaka 565-0871, Japan

2 Medical Imaging Center for Translational Research, Osaka University Graduate School of Medicine, Suita, Osaka, Japan

3 Department of Molecular Imaging in Medicine, Osaka University Graduate School of Medicine, Suita, Osaka, Japan

4 Osaka University Hospital, Suita, Osaka, Japan

5 Osaka Prefectural University, Sakai, Osaka, Japan

6 Immunology Frontier Research Center, Osaka University, Suita, Osaka, Japan tissues/tumors were also estimated from the ${ }^{18} \mathrm{~F}$-FBPA PET images using the following formula: estimated absolute boron concentration $(\mathrm{ppm})=0.0478 \times[\mathrm{BPA}$ dose $(\mathrm{mg} /$ $\mathrm{kg})] \times$ SUV. The measured absolute boron concentrations $(\mathrm{mBC})$ by ICP-OES and the estimated boron concentrations (eBC) from the PET images were compared.

Results The percent difference between the $\mathrm{mBC}$ and $\mathrm{eBC}$ calculated based on the $\mathrm{SUV}_{\max }$ was $-5.2 \pm 21.1 \%$ for the blood, $-9.4 \pm 22.3 \%$ for the brain, $1.6 \pm 21.3 \%$ for the liver, $-14.3 \pm 16.8 \%$ for the spleen, $-9.5 \pm 27.5 \%$ for the pancreas, and $3.4 \pm 43.2 \%$ for the tumor. Relatively large underestimation was observed for the lung $(-48.4 \pm 16.2 \%)$, small intestine $(-37.8 \pm 19.3 \%)$ and large intestine $(-33.9 \pm 11.0 \%)$, due to the partial volume effect arising from the air or feces contained in these organs. In contrast, relatively large overestimation was observed for the kidney $(34.3 \pm 29.3 \%)$, due to the influence of the high uptake in urine.

Conclusions The absolute boron concentrations in tissues/tumors can be estimated from the SUVs on ${ }^{18}$ F-FBPA PET using a practical formula. Caution must be exercised in interpreting the estimated boron concentrations in the lung, small intestine and large intestine, to prevent the adverse effects of overexposure, which could occur due to underestimation by partial volume effect using PET.

Keywords FBPA - Positron emission tomography · Boron concentration

\section{Introduction}

Boron neutron capture therapy (BNCT) is an effective treatment method for recurrent glioma, malignant melanoma, and various other head and neck cancers [1-3]. 
4-borono-phenylalanine (BPA) is used as the major carrier of boron-10 $\left({ }^{10} \mathrm{~B}\right)$ in BNCT. 4-borono-2- ${ }^{18} \mathrm{~F}$-fluorophenylalanine $\left({ }^{18} \mathrm{~F}\right.$-FBPA) $\mathrm{PET}$ is usually performed before BNCT and the accumulations in the tumor and normal tissues are evaluated by determining the relative uptake ratios [4]. If the tumor to normal tissue ( $\mathrm{T} / \mathrm{N}$ ratio) or tumor to blood (T/B ratio) is more than 2.5 , BNCT is considered to be indicated [5]. However, to prevent the adverse effects of the alpha-particles emitted from ${ }^{10} \mathrm{~B}$ after neutron capture, estimation of the absolute boron concentrations in the normal tissues is essential. Our previous study reported the feasibility of estimating the ${ }^{10} \mathrm{~B}$ concentrations in normal organs based on ppm in ${ }^{18} \mathrm{~F}$-FBPA PET before neutron irradiation in BNCT [6]. However, simplification of the calculation method and validation of the method of estimation of the ${ }^{10} \mathrm{~B}$ concentration from PET images are essential before it can be applied in routine clinical practice. The purpose of this study was to establish a practical method to estimate the absolute boron concentrations in tissues after administration of BPA based on the standardized uptake values (SUVs) determined from ${ }^{18} \mathrm{~F}$ FBPA PET images as well as to validate the accuracy of the method.

\section{Methods}

Male F344 rats $(n=7$, body weight $241.7 \pm 28.0 \mathrm{~g}$, 11-13 weeks old) were purchased from Charles River Japan, Inc. (Atsugi, Japan). A rat glioma C6 cell line, derived from gliomas induced by $N$-nitrosomethylurea, was provided by the RIKEN BRC (Tsukuba, Japan). Rat xenograft models of C6 glioma were evaluated 3 weeks after the tumor implantation in the subcutaneous region of either side [7]. All animal experiments were performed in compliance with the guidelines of the Institute of Experimental Animal Sciences. The study protocol was approved by the Animal Care and Use Committee of the Osaka University Graduate School of Medicine (Approval Number: 20-144-008).

${ }^{18}$ F-FBPA was prepared as described previously, using an F-1 synthesizer (Sumitomo Heavy Industries, Tokyo, Japan) [7]. PET/CT data were acquired with a small-animal PET system (Inveon PET/CT system, Siemens Medical Solutions) [8]. PET scanning was performed 60 min after intravenous injection of ${ }^{18} \mathrm{~F}$-FBPA $(30.5 \pm 0.7 \mathrm{MBq})$ into the animals under isoflurane anesthesia ( $2 \%$ plus oxygen). CT was performed before or after the PET acquisition. All PET data were reconstructed using 2-dimensional orderedsubset expectation maximization (16 subsets, 4 iterations), with attenuation and scatter correction. Regional uptake of radioactivity was decay-corrected for the injection time and expressed as the SUV corrected for the injected dose
$(\mathrm{MBq})$ and body weight $(\mathrm{g})$. Volumes of interest (VOIs) were placed in the brain, lung, liver, spleen, pancreas, small intestine, large intestine, kidney, tumor, and blood pool in the left ventricle on the PET images with reference to the CT images, using the PMOD software (Ver. 3.404).

After the PET scanning, BPA-fructose $(167.32 \pm 18.65 \mathrm{mg} / \mathrm{kg})$ was administered by slow intravenous injection over $2 \mathrm{~min}$ to the same subjects. Rats were killed $60 \mathrm{~min}$ after the BPA injection and tissue samples were collected from the major organs and tumors. The absolute boron concentrations (unit: ppm) in the samples were measured by inductively coupled plasma optical emission spectrometry (ICP-OES) [9].

Boron concentrations in the major organs/tumor were also estimated from the ${ }^{18}$ F-FBPA PET images using the following formula.

\section{Estimated boron concentration ( $\mathrm{ppm})$ $=0.0478 \times[\mathrm{BPA}$ dose $(\mathrm{mg} / \mathrm{kg})] \times \mathrm{SUV}$.}

The factor of 0.0478 was derived from the molecular weight ratio of boron to BPA (calculated as 10/209.01). The BPA dose $(\mathrm{mg} / \mathrm{kg})$ was defined as the injected BPA dose $(\mathrm{mg})$ per unit body weight $(\mathrm{kg})$. SUV was calculated as radioactivity measured by $\mathrm{PET}(\mathrm{Bq} / \mathrm{g})$ divided by injected radioactivity per body weight $(\mathrm{Bq} / \mathrm{g})$, where the specific gravity of the tissue was assumed as $1.0(\mathrm{~g} / \mathrm{ml})$. The maximum SUV ( $\left.\mathrm{SUV}_{\text {max }}\right)$ or mean SUV ( $\left.\mathrm{SUV}_{\text {mean }}\right)$ calculated from the VOIs on the ${ }^{18}$ F-FBPA PET images was used for the calculation.

The measured absolute boron concentrations (mBC) by ICP-OES and the estimated boron concentrations (eBC) from the PET images in the major organs and the tumor were compared by a paired $t$ test. The percent differences between the eBC and $\mathrm{mBC}$ were also evaluated and compared between $\mathrm{SUV}_{\text {max }}$-based estimation and $\mathrm{SUV}_{\text {mean }}{ }^{-}$ based estimation. Statistical analyses were carried out using the SPSS, version 19.0 (SPSS, Chicago, IL, USA), and probability values of less than 0.05 were considered to denote statistical significance.

\section{Results}

Comparisons between the $\mathrm{mBC}$ and $\mathrm{eBC}$ values are shown in Table 1. The eBC values calculated from the $\mathrm{SUV}_{\text {mean }}$ were significantly smaller than the $\mathrm{mBC}$ values for the blood, brain, lung, liver, spleen, pancreas, small intestine, large intestine, and the tumor. The eBC values calculated from the $\mathrm{SUV}_{\max }$ were also significantly smaller than the $\mathrm{mBC}$ values for the lung, small intestine and large intestine, and significantly larger for the kidney. The percent differences between the $\mathrm{mBC}$ and eBC based on $\mathrm{SUV}_{\max }$ calculation were $-5.2 \pm 21.1 \%$ for the blood, 
Table 1 Comparison between measured and calculated boron concentrations (ppm) and percent difference between the $\mathrm{mBC}$ and $\mathrm{eBC}(\%)$

\begin{tabular}{lccccc}
\hline & $\begin{array}{l}\text { mBC by ICP- } \\
\text { OES }(\mathrm{ppm})\end{array}$ & $\begin{array}{l}\mathrm{eBC} \text { from } \\
\mathrm{SUV}_{\max }(\mathrm{ppm})\end{array}$ & $\begin{array}{l}\mathrm{eBC} \text { from } \\
\mathrm{SUV}_{\text {mean }}(\mathrm{ppm})\end{array}$ & $\begin{array}{l}\text { Difference between mBC } \\
\text { and eBC from SUV }\end{array}$ max $(\%)$ & $\begin{array}{l}\text { Difference between mBC } \\
\text { and eBC from SUV }\end{array}$ \\
\hline Blood & $12.2 \pm 1.0$ & $11.6 \pm 2.7$ & $9.0 \pm 1.9^{* *}$ & $-5.2 \pm 21.1$ & $-26.6 \pm 12.6$ \\
Brain & $7.6 \pm 1.1$ & $8.2 \pm 1.3$ & $5.3 \pm 1.3^{* *}$ & $9.4 \pm 22.3$ & $-30.1 \pm 19.0$ \\
Lung & $15.0 \pm 3.9$ & $7.3 \pm 1.0^{* *}$ & $4.9 \pm 1.0^{* *}$ & $-48.4 \pm 16.2$ & $-65.7 \pm 12.1$ \\
Liver & $14.9 \pm 2.4$ & $14.9 \pm 2.6$ & $11.6 \pm 2.3^{*}$ & $1.6 \pm 21.3$ & $-20.8 \pm 18.8$ \\
Spleen & $20.4 \pm 4.7$ & $16.9 \pm 2.0$ & $11.0 \pm 1.5^{* *}$ & $-14.3 \pm 16.8$ & $-44.0 \pm 13.3$ \\
Pancreas & $52.2 \pm 12.1$ & $46.2 \pm 12.1$ & $31.2 \pm 7.0^{* *}$ & $-9.5 \pm 27.5$ & $-37.4 \pm 23.6$ \\
Small intestine & $16.4 \pm 2.5$ & $9.9 \pm 1.9^{* *}$ & $6.9 \pm 1.5^{* *}$ & $-37.8 \pm 19.3$ & $-56.7 \pm 11.8$ \\
Large intestine & $13.2 \pm 1.5$ & $8.7 \pm 1.6^{* *}$ & $5.8 \pm 1.4^{* *}$ & $-33.9 \pm 11.0$ & $-55.4 \pm 11.8$ \\
Kidney & $83.1 \pm 16.1$ & $108.9 \pm 17.1^{*}$ & $89.1 \pm 14.5$ & $34.3 \pm 29.3$ & $10.0 \pm 24.9$ \\
Tumor & $27.6 \pm 8.5$ & $26.0 \pm 6.0$ & $15.2 \pm 3.4^{* *}$ & $3.4 \pm 43.2$ & $-42.4 \pm 16.5$ \\
\hline
\end{tabular}

** $p<0.01$ and $* p<0.05$ by paired $t$ test

$-9.4 \pm 22.3 \%$ for the brain, $1.6 \pm 21.3 \%$ for the liver, $-14.3 \pm 16.8 \%$ for the spleen, $-9.5 \pm 27.5 \%$ for the pancreas, and $3.4 \pm 43.2 \%$ for the tumor. Relatively large underestimation was observed for the lung $(-48.4 \pm 16.2 \%)$, small intestine $(-37.8 \pm 19.3 \%)$ and large intestine $(-33.9 \pm 11.0 \%)$, possibly due to the partial volume effect arising from the air or feces contained in these organs. In contrast, large overestimation was observed for the kidney $(34.3 \pm 29.3 \%)$ due to the influence of the high uptake in urine.

\section{Discussion}

Our previous study demonstrated the existence of a significant positive correlation between the accumulation levels of BPA and ${ }^{18}$ F-FBPA [9]. In the present study, we demonstrated the feasibility of estimating the absolute boron concentrations in tissues/tumors after administration of BPA based on the SUVs determined from ${ }^{18} \mathrm{~F}$-FBPA PET images, which also showed good correlations between the boron concentration and the ${ }^{18}$ F-FBPA uptake, consistent with our previous report (Supplemental Fig. 1). Furthermore, we found that more accurate estimation was afforded by the $\mathrm{SUV}_{\text {max }}$ than by the $\mathrm{SUV}_{\text {mean }}$, except for the case of the kidney. SUV $\mathrm{max}_{\text {max }}$ is a major, frequently used index in clinical oncology practice; therefore, the method described herein can easily be applied in routine clinical practice.

Estimation of the absolute boron concentrations in normal tissues is important, because alpha-particles from ${ }^{10} \mathrm{~B}$ show large energy transfer and have the potential to cause severe adverse effects in the event of overexposure of the neutron beam [10]. Careful estimation is essential, especially when BNCT is applied for lung or abdominal cancers. Relatively large underestimation was observed for the lung, small intestine and large intestine, due to the partial volume effect.
Caution is needed, because underestimation of the tissue boron concentration might lead to excessive radiation exposure of the corresponding tissues. In contrast, large overestimation was observed for the kidney $(34.3 \pm 29.3 \%)$; the percent difference for the kidney was $10.0 \pm 24.9 \%$ in the $\mathrm{SUV}_{\text {mean-based estimation, which is considered to be optimal }}$ for the kidney. In the tumors, the relationship between $\mathrm{mBC}$ and $\mathrm{eBC}$ depends on the intratumoral heterogeneity of $\mathrm{C6}$ glioma. Since we measured the whole tumor content for the measurement of boron concentration by ICP-OES, mBC showed smaller values with the increase of necrotic regions. Whereas, eBC from SUVmax reflected the hottest region of the tumor, which showed a certain degree of variability (Supplemental Fig. 2). As a result, standard deviation of the percent difference in the tumor $(43.2 \%)$ was relatively large as compared to that for the major organs.

We recently reported about the intratumoral heterogeneity with hypoxic and necrotic regions in the C6 glioma xenograft by comparing ${ }^{18}$ F-FMISO and ${ }^{15} \mathrm{O}$-labeled gas PET to histological analysis [11]. Another previous study reported the characteristic difference in the tumor xenograft by comparing three tumor cell lines [12]. In that study, U251 (human glioblastoma) xenograft showed the necrotic region with the minimal stromal component, whereas BxPC-3 (human pancreatic ductal adenocarcinomas) showed abundant stroma and no apparent necrotic region. Tumor-associated stroma is the key determinant for the tumor hypoxia and necrosis which is related to the intratumoral heterogeneity.

Our previous study demonstrated the feasibility of estimating the tissue/tumor boron concentration using ${ }^{18} \mathrm{~F}$ FBPA PET in humans [6]. In the present study, we simplified the estimation method using SUV and validated its accuracy by comparing the results with the actually measured boron concentrations. The estimated image map of boron concentration (unit: ppm) can be obtained from the 
PET images (unit: SUV) by simply multiplying with a coefficient, calculated as $0.0478 \times$ [BPA dose $(\mathrm{mg} / \mathrm{kg})]$.

In this study, we used the molecular weight of BPA as a simple substance, not as the complexed compound. BPA complexed solutions, such as BPA-fructose or BPA-mannitol, are usually used for intravenous infusion because of the low solubility of BPA at physiological pH [13, 14]. If the molecular weight of BPA-fructose is applied, coefficient is calculated as $0.0259 \times$ [BPA-fructose dose $(\mathrm{mg} /$ $\mathrm{kg})]$ in the formula. However, fructose and mannitol are solubilizing agents, and there is room for further optimization. The infusion dose of BPA $(\mathrm{mg} / \mathrm{kg})$ should be normalized using the weight of the simple BPA compound for accurate comparisons among studies.

A limitation of our study is related to the administration method of BPA. We used slow bolus injection of BPA over 2 min, similar to that for ${ }^{18}$ F-FBPA injection. However, continuous infusion of BPA is often used in the clinical procedure of BNCT [15]. For practical use for clinical setting of BNCT, further study with comparing $\mathrm{mBC}$ by drip infusion and eBC might be needed.

\section{Conclusions}

The absolute boron concentrations in tissues/tumors can be estimated from the SUVs determined by ${ }^{18}$ F-FBPA PET using a simple formula, suggesting the feasibility of this method in clinical practice. However, caution must be exercised in interpreting the estimated boron concentrations in the lung, small intestine and large intestine to prevent the adverse effects of neutron beam overexposure, which could arise as a result of underestimation by partial volume effect using PET.

Acknowledgements We would like to thank the staff of the PET drug synthesis department of the Osaka University Hospital for the preparation of ${ }^{18}$ F-FBPA, Genki Horitsugi, Keiko Matsunaga, Kayako Isohashi, Hiroki Kato, and Mitsuaki Tatsumi for their excellent assistance. This study was supported by a Grant (No. 24591758) from the Ministry of Education, Culture, Sports, Science and Technology, Japan. No potential conflicts of interest were disclosed.

\section{Compliance with ethical standards}

Funding This study was funded by KAKENHI Grant-in-Aid for Scientific Research (S) (No. 24229008) and (A) (No. 24591758) from the Ministry of Education, Culture, Sports, Science and Technology (MEXT), Japan.

Open Access This article is distributed under the terms of the Creative Commons Attribution 4.0 International License (http://crea tivecommons.org/licenses/by/4.0/), which permits unrestricted use, distribution, and reproduction in any medium, provided you give appropriate credit to the original author(s) and the source, provide a link to the Creative Commons license, and indicate if changes were made.

\section{References}

1. Nariai T, Ishiwata K, Kimura Y, Inaji M, Momose T, Yamamoto $\mathrm{T}$, et al. PET pharmacokinetic analysis to estimate boron concentration in tumor and brain as a guide to plan BNCT for malignant cerebral glioma. Appl Radiat Isot. 2009;67(7-8 Suppl):S348-50. doi:10.1016/j.apradiso.2009.03.061.

2. Ishiwata K, Ido T, Honda C, Kawamura M, Ichihashi M, Mishima Y. 4-Borono-2- $\left[{ }^{18}\right.$ F]fluoro-D, L-phenylalanine: a possible tracer for melanoma diagnosis with PET. Int J Radiat Appl Instrum B. 1992;19(3):311-8.

3. Kato I, Fujita Y, Maruhashi A, Kumada H, Ohmae M, Kirihata $\mathrm{M}$, et al. Effectiveness of boron neutron capture therapy for recurrent head and neck malignancies. Appl Radiat Isot. 2009;67(7-8 Suppl):S37-42. doi:10.1016/j.apradiso.2009.03.103.

4. Isohashi K, Shimosegawa E, Naka S, Kanai Y, Horitsugi G, Mochida I, et al. Comparison of the image-derived radioactivity and blood-sample radioactivity for estimating the clinical indicators of the efficacy of boron neutron capture therapy (BNCT): 4-borono-2- ${ }^{18}$ F-fluoro-phenylalanine (FBPA) PET study. EJNMMI Res. 2016;6(1):75. doi:10.1186/s13550-016-0230-7.

5. Aihara T, Morita N, Kamitani N, Kumada H, Ono K, Hiratsuka J, et al. Boron neutron capture therapy for advanced salivary gland carcinoma in head and neck. Int $J$ Clin Oncol. 2014;19(3):437-44. doi:10.1007/s10147-013-0580-3.

6. Shimosegawa E, Isohashi K, Naka S, Horitsugi G, Hatazawa J. Assessment of 10B concentration in boron neutron capture therapy: potential of image-guided therapy using ${ }^{18}$ FBPA PET. Ann Nucl Med. 2016;30(10):749-55. doi:10.1007/s12149-016-11218 .

7. Watabe T, Ikeda H, Nagamori S, Wiriyasermkul P, Tanaka Y, Naka S, et al. ${ }^{18}$ F-FBPA as a tumor-specific probe of L-type amino acid transporter 1 (LAT1): a comparison study with ${ }^{18} \mathrm{~F}$ FDG and 11C-methionine PET. Eur J Nucl Med Mol Imaging. 2017;44(2):321-31. doi:10.1007/s00259-016-3487-1.

8. Bao Q, Newport D, Chen M, Stout DB, Chatziioannou AF. Performance evaluation of the inveon dedicated PET preclinical tomograph based on the NEMA NU-4 standards. J Nucl Med. 2009;50(3):401-8. doi:10.2967/jnumed.108.056374.

9. Hanaoka K, Watabe T, Naka S, Kanai Y, Ikeda H, Horitsugi G, et al. FBPA PET in boron neutron capture therapy for cancer: prediction of (10)B concentration in the tumor and normal tissue in a rat xenograft model. EJNMMI Res. 2014;4(1):70. doi:10. 1186/s13550-014-0070-2.

10. Monti Hughes A, Pozzi E, Thorp SI, Curotto P, Medina VA, Martinel Lamas DJ, et al. Histamine reduces boron neutron capture therapy-induced mucositis in an oral precancer model. Oral Dis. 2015;21(6):770-7. doi:10.1111/odi.12346.

11. Watabe T, Kanai Y, Ikeda H, Horitsugi G, Matsunaga K, Kato H, et al. Quantitative evaluation of oxygen metabolism in the intratumoral hypoxia: ${ }^{18} \mathrm{~F}$-fluoromisonidazole and ${ }^{15} \mathrm{O}$-labelled gases inhalation PET. EJNMMI Res. 2017;7(1):16. doi:10.1186/ s13550-017-0263-6.

12. Koyasu S, Tsuji Y, Harada H, Nakamoto Y, Nobashi T, Kimura $\mathrm{H}$, et al. Evaluation of tumor-associated stroma and its relationship with tumor hypoxia using dynamic contrast-enhanced CT and ${ }^{(18)} \mathrm{F}$ misonidazole PET in murine tumor models. Radiology. 2016;278(3):734-41. doi:10.1148/radiol.2015150416.

13. Cruickshank GS, Ngoga D, Detta A, et al. A cancer research UK pharmacokinetic study of BPA-mannitol in patients with high grade glioma to optimise uptake parameters for clinical trials of BNCT. Appl Radiat Isot. 2009;67(7-8 Suppl):S31-3.

14. Heikkinen $S$, Savolainen $S$, Melkko P. In vitro studies on stability of L-p-boronophenylalanine-fructose complex (BPA-F). J Radiat Res. 2011;52(3):360-4. 
15. Watanabe T, Hattori $\mathrm{Y}$, Ohta $\mathrm{Y}$, Ishimura $\mathrm{M}$, Nakagawa $\mathrm{Y}$, Sanada Y, et al. Comparison of the pharmacokinetics between LBPA and L-FBPA using the same administration dose and protocol: a validation study for the theranostic approach using $\left[{ }^{18} \mathrm{~F}\right]-$
L-FBPA positron emission tomography in boron neutron capture therapy. BMC Cancer. 2016;16(1):859. doi:10.1186/s12885-0162913-x. 